\title{
Assessing good quality dental care
}

As patients, we want the dental care necessary to ensure that we are free from pain, able to eat, speak, smile, laugh, and kiss without embarrassment. We also want advice about self care. Many of these outcomes are based on aesthetic and social norms which are defined by each individual and hence can be judged by people themselves. What dentists can do to contribute to these ordinary ends involves the manipulation of increasingly extraordinary materials and equipment in ways that are invisible to the patient. It is this apparent paradox that assessments of quality in dental care have to address.

These distinctive and diverse characteristics mean that the practice of "good" dentistry is never easy. The two articles in this copy of the journal provide some useful building blocks.

Consider first the technical evaluations. As in medicine, ${ }^{1}$ many dentists depend on manufacturers' assessments of materials - assessments often skewed to promote novelty and consumption and not based on effectiveness criteria. The effectiveness bulletin published on pages $202-7$ is therefore a welcome addition. It deals with amalgam restorations, one of the most common dental treatments. It will help dentists and patients to resist inappropriate commercial pressure to replace the more cost effective amalgam fillings with newer more expensive "white" filling materials.

The authors note that around $60 \%$ of all restorative dentistry involves replacement of existing restorations and that this has more to do with the variation in dentists' decision making than variation in disease processes or materials' performance characteristics. Perhaps one way to bridge the gap between technology assessments and service user concerns might be to set standards for the longevity of restorations. A patient might reasonably want to know the expected life of a given type of restoration, say in terms of " $50 \%$ of two surface fillings last at least $\mathrm{x}$ months/years". The extensive literature cited in the review would allow us to do this now.

The second article (page 149) has a different starting point for assessing quality in dental care, namely public and user perspectives. As one would expect, people's wants of general dental care are similar to their wants of other health services. It is a useful reminder that people care as much about how they are treated as people as they do about the way their condition/disease is treated.

Politicians and policy makers would do well to note the finding that satisfaction has declined as general dental services have drifted from indirect NHS funding to direct patient payments. This is just one of the many insights which could be gained from the general dental serices as a "natural experiment" in alternative funding mechanisms. These include fee per item payments as incentives for productivity and their effect on increasing supply without improving outcomes; the use of private capital (general dental practitioners do not have access to public funds for premises or equipment) and its unintended consequences such as increasing inequity of provision; and creeping privatisation and its impact on both the quality of and access to health care.

The authors draw our attention to the greater priority people give to technical skill and pain relief in comparison with other forms of primary care provision. The first concern relates to the significant role of the use of technology in dental practice referred to earlier. The second concern relates to the peculiar challenge faced by dentists. They, unusually among primary care practitioners and indeed many secondary care practitioners, perform invasive interventions on conscious patients. In this, their practice is more akin to day case surgery than consultations in general medical care. This has profound implications for definitions of quality dental care. To respond to users' concerns we may have to look beyond the usual accessibility targets to include indicators of the communication skills which we expect of dentists in settings that provoke anxiety.

The views of dentists and lay people should be used to develop a set of standards for both processes of care and outcomes which can shape what we expect of each other in producing reasonable standards of care.

DIANE PLAMPING

Urban Partnerships Group, Department of Operational Research, London School of Economics

1 Griffith D. Reasons for not seeing drug representatives. BMF 1999;319:6970 . 